\title{
Heartbeat: The worldwide burden of atrial fibrillation
}

Atrial fibrillation (AF) has emerged as one of the few cardiovascular diseases in which both the number of cases and the age-adjusted prevalence has increased over the last decades in developed countries, leading to a substantial economic burden. Most data on AF prevalence and outcomes comes from western countries. However, Kim and colleagues ${ }^{1}$ present an analysis of recent trends in the hospital-care burden of AF in an Asian country - South Korea. Using the National Health Insurance Service (NHIS) database, this nationwide cohort included 931138 patients hospitalised for AF from 2006 and 2015. There was an overall trend towards an increase in AF hospitalisation from 767 to 3986 per 1 million Koreans (relative increase, $420 \%$ ) over this time period. This increase in AF hospitalisation was observed in all age, sex and economic status sub-groups. The frequency of comorbidities and mean CHA2DS2-VASc and HAS-BLED scores also increased continuously during this period, as well as hospitalizations due to complications of both the $\mathrm{AF}$ and of its treatment, specifically major bleeding (figure 1). Mortality associated with AF hospitalizations decreased from $7.5 \%$ in 2006 to $4.3 \%$ in 2015 (relative decrease $42 \%$ ), although hospital costs per year increased exponentially by $468 \%$ during this 10 year period. Considering that the length of hospital stay is relatively long in Korea, the authors conclude that "prevention of AF hospitalisations and streamlined integrated AF management should be pursued in a holistic manner to lessen the healthcare burden of AF'.

In a companion editorial, Patel and colleagues $^{2}$, discuss this study in the context of the global rising trends of $\mathrm{AF}$ prevalence. 'This underscores the urgent need for healthcare systems around the world to develop and implement programmes for preventing AF and new paradigms to optimise identification and treatment of patients with AF.'

There is an increased risk of heart failure in patients with rheumatoid

\footnotetext{
'Internal Medicine, Universidade Federal de Minas Gerais, Belo Horizonte, Brazil

${ }^{2}$ Division of Cardiology, University of Washington, Seattle, Washington, USA
}

Correspondence to Professor Catherine M Otto, Division of Cardiology, University of Washington, Seattle,WA 98195, USA; cmotto@uw.edu

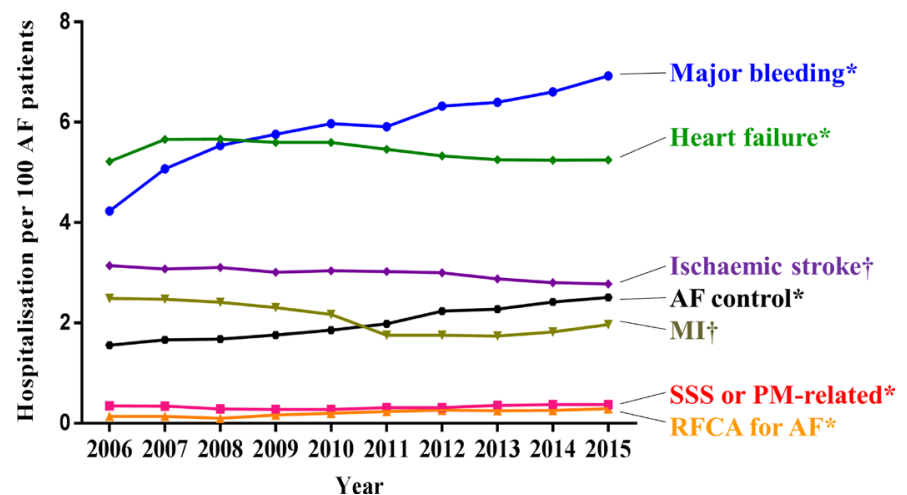

Figure 1 Temporal trends of AF hospitalisation per 100 patients with AF according to main hospitalisation causes between 2006 and 2015. ${ }^{*} P$ value for increase trends $<0.001$. $+P$ value for decrease trends $<0.001$. AF, atrial fibrillation; MI, myocardial infarction; PM, pacemaker; RFCA, radiofrequency catheter ablation; SSS, sick sinus syndrome. arthritis, presumably related to a chronic inflammatory state, yet the mechanisms of this association have not been well studied. In a mouse model of rheumatoid arthritis, Pironti and colleagues ${ }^{3}$ demonstrated adverse cardiac remodelling with hypertrophy, fibrosis and reduced ventricular systolic function. At the molecular level, they found dysregulation of cardiomyocyte cytosolic and sarcoplasmic reticulum calcium $\left(\mathrm{Ca}^{2+}\right)$ signalling pathways, concluding that " $\mathrm{Ca}^{2+}$ handling proteins displayed oxidation-dependent posttranslational modifications that together with an increase in superoxide dismutase expression indicate a cell environment with oxidative stress' (figure 2).

In an editorial, Del Buono, Abbate and Toldo ${ }^{4}$ remind us that patients with rheumatoid arthritis are at increased risk of cardiovascular death from both atherosclerotic disease and heart failure. In addition to conventional risk factors, 'A combined interaction of cytokine spillover, oxidative

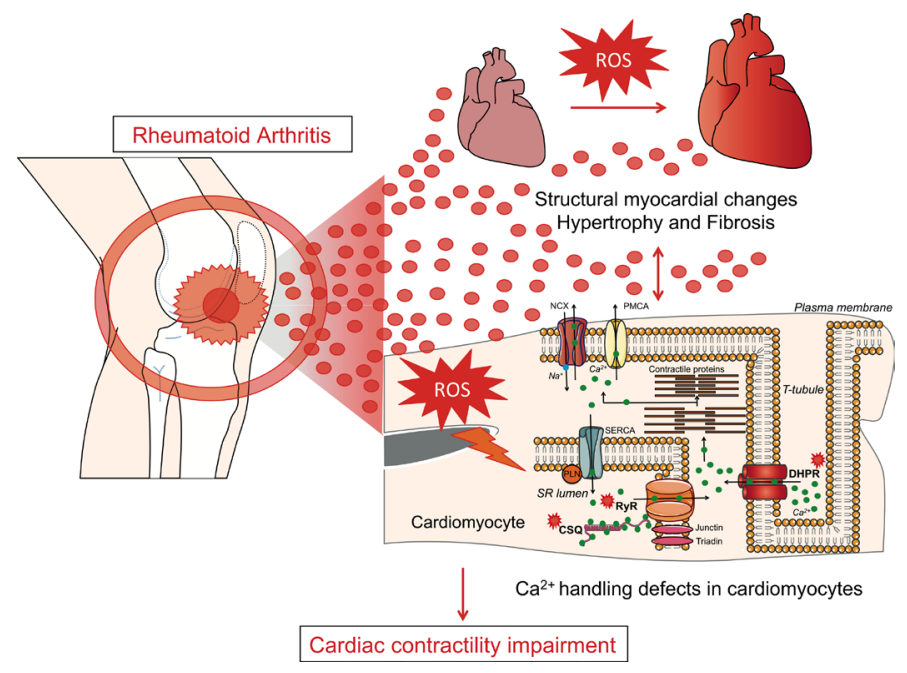

Figure 2 Cartoon depicting cardiac remodelling in experimental rheumatoid arthritis (RA). RA leads to structural and functional remodelling of the heart consisting of cardiac hypertrophy and fibrosis coupled to impaired contractile function. In cardiomyocytes, high levels of reactive oxygen species (ROS) promote post-translational modification of important $\mathrm{Ca}^{2+}$ handling proteins (RyR2, DHPR and CSQ). This was linked to impaired $\mathrm{Ca}^{2+}$ handing with reduced L-type $\mathrm{Ca}^{2+}$ current, SR $\mathrm{Ca}^{2+}$ stores and peak $\mathrm{Ca}^{2+}$ transient amplitudes and reduced cardiomyocyte contractility. CSQ, calsequestrin; DHPR, dihydropyridine receptor; RyR2, ryanodine receptor 2. 


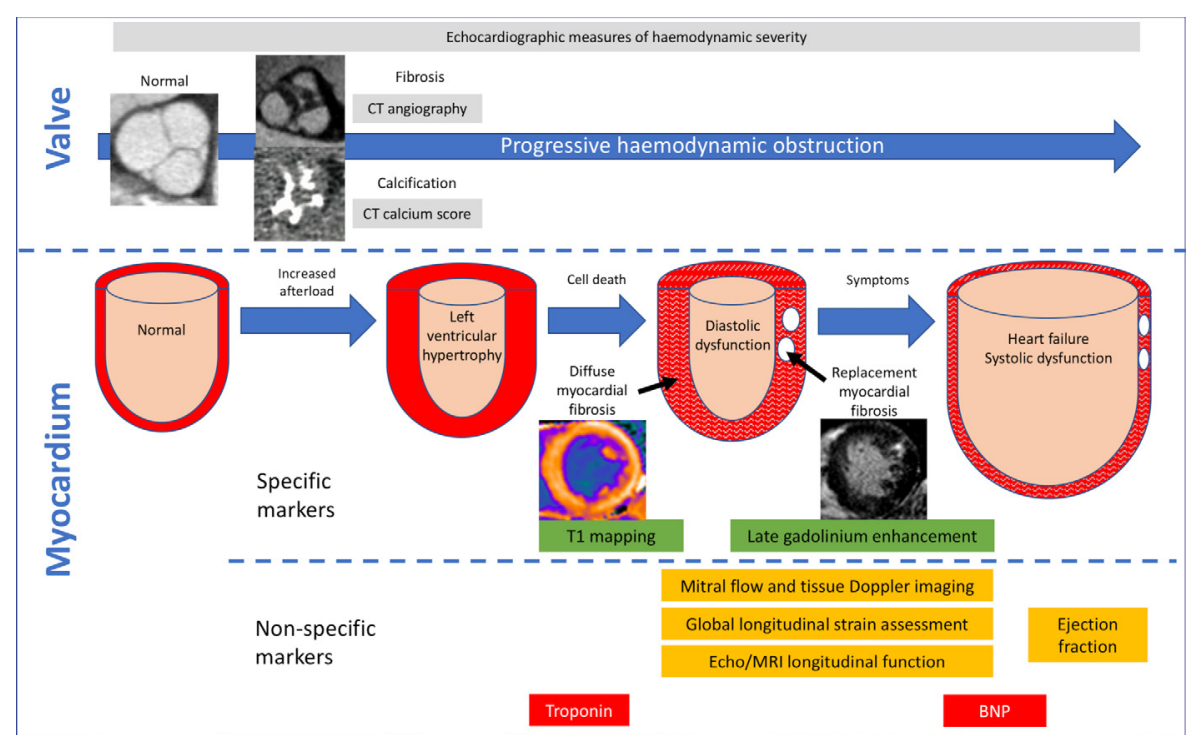

Figure 3 Imaging and biomarker assessments of stage of valvular stenosis and myocardial response to increased afterload. Progressive haemodynamic obstruction as a result of aortic leaflet restriction is assessed using echocardiography. However, specific valvular pathologies such as fibrosis and calcification can be assessed using CT methods. Ejection fraction is a poorly sensitive marker of myocardial decompensation with abnormalities in Doppler measures, longitudinal strain and systolic function, which are all detectable prior to this. However, these measures, along with biomarkers such as troponin and B-type natriuretic peptide (BNP) are non-specific and may be abnormal as a result of coexistent myocardial pathology such as coronary heart disease. T1 mapping methods and late gadolinium enhancement are more specific for decompensation as a result of pressure overload.

stress, abnormal innate and specific immune adaptation and coagulation abnormalities have been considered as potential mechanisms of increased cardiovascular risk.' Given the complexities in unravelling the mechanisms of disease in patients with rheumatoid arthritis, they suggest that this 'mouse model is therefore a useful tool to study simultaneously changes in the cardiovascular system and heart function and the joints and how these systems/organs respond to targeted therapies. This may be particularly important because therapies that improve arthritis may show an unfavourable cardiovascular safety profile.'

In a study of how a BMJ Learning training module improved interpretation of ECG tracings in athletes, Riding and Drezner ${ }^{5}$ found that ECG interpretation was much more consistent and accurate after training, despite significant baseline differences depending on medical specialty. Research into educational approaches, such as demonstrated in this study, is important to improve the efficacy and efficiency of medical training; which ultimately translates into better care of our patients and improved population health. As the authors conclude: "With the evolving criteria for ECG interpretation eliciting ever improving levels of specificity and sensitivity in the detection of conditions associated with sudden cardiac death among athletes, training is required to ensure the infrastructure and personnel is in place to uphold these standards. The BMJ Learning course presented is a valuable first step and demonstrates that such an online tool can be effective in aiding ECG interpretation among healthcare professionals globally.'

In the Education in Heart article in this issue, Everett, Clavel, Pibarot and Dweck $^{6}$ provide a updated summary of the optimal timing of intervention in adults with aortic valve stenosis. As our options for intervention have expanded beyond surgical aortic valve replacement to include a transcatheter bioprosthetic valve, decision making has become more complex. It is especially important to offer intervention 'just as left ventricular decompensation is starting to develop' and not too early, given the limited durability of bioprosthetic valves, and not too late, given the irreversible changes and futility of intervention at that point in the disease course (figure 3).

The Image Challenge question ${ }^{7}$ asks you to interpret multi-modality images in a 52 year old women with ventricular tachycardia. The answer might surprise you.

\section{Competing interests None declared.}

Provenance and peer review Commissioned; internally peer reviewed.

(C) Author(s) (or their employer(s)) 2018. No commercial re-use. See rights and permissions. Published by BMJ.

$$
\text { A) Check for updates }
$$

To cite Ribeiro AL, Otto CM. Heart 2018;104:1987-1988.

Heart 2018:104:1987-1988.

doi:10.1136/heartjnl-2018-314443

\section{REFERENCES}

1 Kim D, Yang PS, Jang E, et al. Increasing trends in hospital care burden of atrial fibrillation in Korea, 2006 through 2015. Heart 2018;104:2010-17.

2 Patel NJ, Atti V, Mitrani RD, et al. Global rising trends of atrial fibrillation: a major public health concern. Heart 2018:104:1989-90.

3 Pironti G, Bersellini-Farinotti A, Agalave NM, et al. Cardiomyopathy, oxidative stress and impaired contractility in a rheumatoid arthritis mouse model. Heart 2018;104:2026-34

4 Del Buono M, Abbate A, Toldo S. Interplay of inflammation, oxidative stress and cardiovascular disease in rheumatoid arthritis. Heart 2018;104:1991-2.

5 Riding NR, Drezner JA. Performance of the BMJ learning training modules for ECG interpretation in athletes. Heart 2018;104:2051-7.

6 Everett RJ, Clavel MA, Pibarot P, et al. Timing of intervention in aortic stenosis: a review of current and future strategies. Heart 2018;104:2067-76.

7 Fidalgo A, Fernandez-Friera L, Solis J. A 52-year-old woman with ventricular tachycardia. Heart 2018;104:2025. 